\title{
AGENT-BASED MODEL OF AUFTRAGSTAKTIK: SELF ORGANIZATION IN COMMAND AND CONTROL OF FUTURE COMBAT FORCES
}

\author{
Robert H. Kewley, Jr \\ Center for Army Analysis \\ 6001 Goethals Road \\ Fort Belvoir, VA 22313, U.S.A.
}

\begin{abstract}
Agent-based modeling is a framework that allows the analysis of distributed command-by-influence using mission-type orders known for over a century as Auftragstaktik in German Army manuals. A combat simulation with embedded decision agents analyzed this type of decentralized command and control. Local commanders relied on their improved situation awareness, mission goals, and constraints from higher commander to drive a small set of robust decision methods. In this sense, they self-organized, and an effective set of actions for mission accomplishment emerged. The improvement was most dramatic for more capable future combat forces. Agent-based modeling provides a laboratory for experiments in command and control of future combat forces.
\end{abstract}

\section{INTRODUCTION}

In the words of Major General Werner Widder, German Army, "Only Auftragstaktik enables the meaningful exploitation of the most sophisticated technology, and only Auftragstaktik allows mastery of the increasingly complex challenges of the 21st century (Widder, 2002)." Auftragstaktik is loosely translated as "mission-oriented tactics" in English. However, as a command and control principle, it involves much more. German Army Regulations further explain, "Auftragstaktik is the pre-eminent command and control principle in the Army. It is based on mutual trust and requires each soldier's unwavering commitment to perform his duty. The military leader informs what his intention is, sets clear achievable objectives, and provides the required forces and resources. He will only order details regarding execution if measures which serve the same objective have to be harmonized, if political or military constraints require it. He gives latitude to subordinate leaders in the execution of their mission (German Army Regulation 100/100, 2002)."

This paper demonstrates a technique by which agentbased modeling simulates the effects of Auftragstaktik on a military mission. Embedding command and control agents with each entity in the model, the combat simulation gains the ability to simulate the effects of tactical decisions made during the fight. A command and control experiment with such a model demonstrated that as military forces become more capable, such as the US Army future forces equipped with the Future Combat System (FCS), there is a much greater payoff for allowing lower level commanders greater freedom of action.

\section{TACTICAL DECISION AGENTS}

Embedding tactical decision agents into a combat model is a technique that allows a combat model to estimate the effects of command and control techniques given certain information available to the force. Agents are programmed software modules that scan their environment and make a decision (Ilachinski, 1996). In a military context, these decisions may be local decisions, such as moving one vehicle to avoid incoming fire, or global decisions such as the allocation of fire missions to a suite of shooters in order to engage the known set of targets. These agents help overcome the difficulty of modeling information effects for future combat forces.

Information itself has little intrinsic value. It becomes an advantage only when superior information leads to a superior tactical decision and a superior tactical action. Future combat forces are expected to leverage an information advantage into superior fire and maneuver. These forces gain information about the enemy and terrain using advanced intelligence, reconnaissance, and surveillance systems. They analyze distribute that information via advanced communications and computer systems. Command and control systems transform that information advantage into a tactical advantage by using the available information to direct tactical actions which yield an advantage over enemy forces. Tactical decision agents allow simulation of these command and control systems.

Unfortunately, most constructive simulations lack the ability to model adaptive command and control methods similar to Auftragstaktik. Frequently, entities in the model move along prescribed routes stopping at prescribed times 
to perform certain actions. This technique requires the analyst to determine in advance the best course of action for all entities in the simulation. Combatants are not permitted to deviate from these plans as new information becomes available during the model run. This modeling paradigm does not allow for simulation of the effects of command and control systems. However, allowing tactical decision agents to acts as surrogates for small unit and even vehicle commanders introduces freedom of action into the simulation. This freedom of action allows for the simulation of the effects of different command and control systems, to include simulation of the effects of Auftragstaktik.

\section{JCOMBAT MODELING ENVIRONMENT}

In order to introduce tactical decision agents into a combat simulation, this project's analysts developed JCombat, a high-resolution, moderately-detailed, but fast-funning combat simulation written in Java. JCombat exists as a test bed for command and control agents for potential integration into even more detailed accredited simulation models. It contains a detailed terrain model and line of sight algorithms. It handles direct and indirect fire in great detail. However, programming time constraints and an interest in execution speed forced a more abstract representation of target acquisition, communications, and intelligence processes (Kewley and Larimer, 2003).

The JCombat simulation contains enough detail to allow the friendly force to receive a set of spot reports, build a partial common operational picture for the friendly force, and disseminate that picture to subordinate units for decision-making. This common operational picture, along with some friendly objectives, was used by each unit in the friendly force to continuously update its routes and positions during the fight to seek a position of advantage and better accomplish unit missions. Three decision agents performed these tasks, the position agent, the route agent, and the vehicle agent.

An operations order in JCombat, instead of specifying an explicit route for a unit, gives the unit a movement technique and allows the route agent to generate the unit's route. This movement technique defines the objectives a unit should seek as it develops its route. For example, the assault movement technique seeks speed and enemy destruction, while the recon technique seeks enemy acquisition as opposed to destruction, with little emphasis on speed. This is accomplished with the aid of a linear value model that assigns weights to the individual tactical characteristics of the route. A genetic algorithm uses the route evaluation model to generate routes which give the unit a tactical advantage in the current tactical situation (Kewley and Larimer, 2003).

A unit may adjust not only its route, but also its final destination, bounded by a flexibility distance specified in the plan. The flexibility distance is the distance that the plan allows a unit to move from its assigned position in or- der to better accomplish its mission. Units in this scenario were assigned a flexibility distance of 2000-3000 meters. In a manner similar to the one used by the route selection agent to evaluate routes, the positioning agent evaluates candidate positions for a mission given by the plan - attack by fire, support by fire, defend, recon, delay, or hide. A mission is a set of objectives sought by the unit when it reaches its destination. For example, both the attack by fire and support by fire missions seek enemy destruction. However, the support by fire mission also places greater emphasis on staying close to the location given by the plan, so that it does not get too far away from the unit whose movement it supports. Since the search for a single position is a much simpler task than the search for a route, the position agent uses a simple uniform random search as opposed to a genetic algorithm (Kewley and Larimer, 2003).

Figure 1 shows the results of a simulation run using both of these unit agents. Friendly forces are the darker symbols between the 19 and 22 east-west grid lines. Enemy forces are the lighter symbols concentrated in the hilly terrain between the 22 and 24 east-west grid lines. Friendly forces move as platoon sized elements in formation along routes which avoid enemy forces until they gain a position of advantage. This allows for more advantageous engagement of enemy force and better survival of friendly forces. However, these unit agents still require platoon-sized units to maintain formation during the fight.

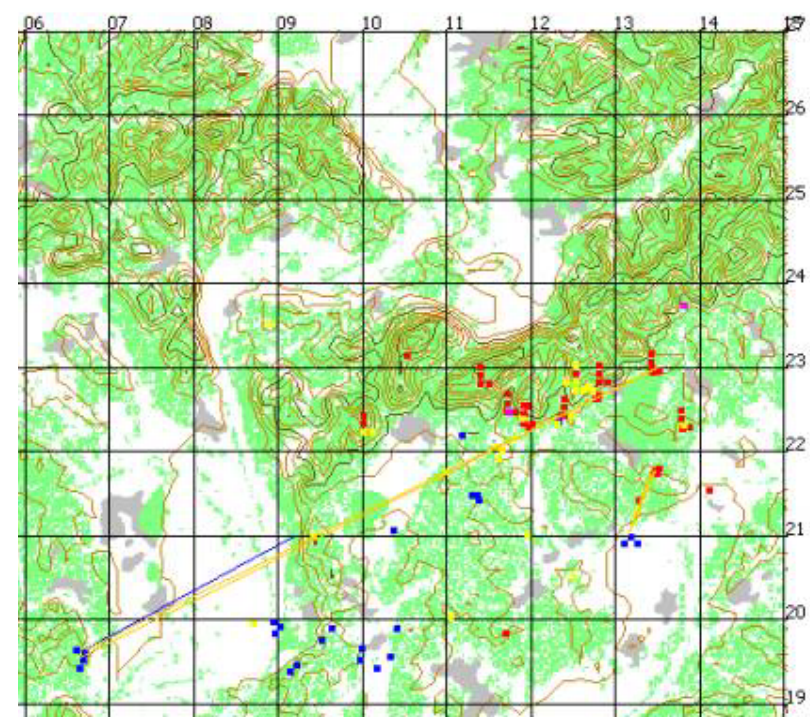

Figure 1: JCombat Simulation with Unit Agents

The vehicle agent allows further freedom of movement for vehicles as well. The position agent assigns a best position to a unit based on mission. The vehicle agent uses a random search technique similar to the position agent to move each vehicle in the unit independently to a position of advantage within a certain distance of the location specified by the position agent. The vehicle agent allows for better dispersal of the vehicles and a more robust search for 
a position of advantage. The force is synchronized by task, purpose, and situation as opposed to synchronized by plan. Figure 2 shows a more dispersed friendly force as it moves using both unit and vehicle agents to engage enemy forces.

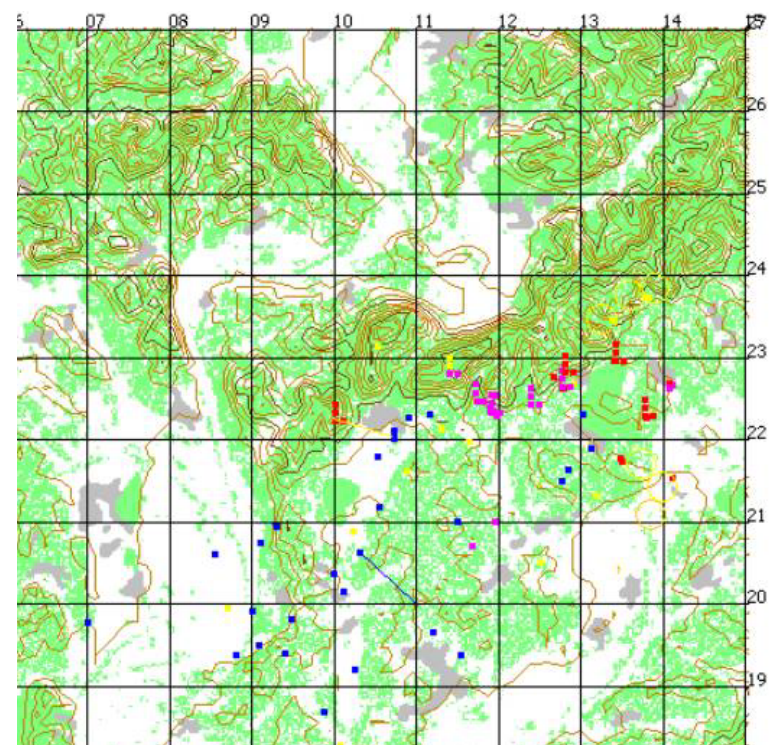

Figure 2: JCombat Simulation with Unit and Vehicle Agents

\section{COMMAND AND CONTROL EXPERIMENTAL DESIGN}

The capabilities of JCombat allow for the design of an experiment to test the effects of different command and control systems. The measures of performance used in this experiment will be friendly losses and enemy losses during combat. The null hypothesis is that the performance of distributed command and control systems, such as Auftragstaktik, is equal to the performance of more centralized command and control systems. The alternative hypothesis is that they are not equal. The experiment tested this hypothesis for both current and future forces. Figure 3 shows the array of friendly and enemy forces for this experiment. Elements of an allied tank-heavy company-team (left) must cross a valley and attack to destroy dispersed and wellhidden elements of a defending enemy force (right).

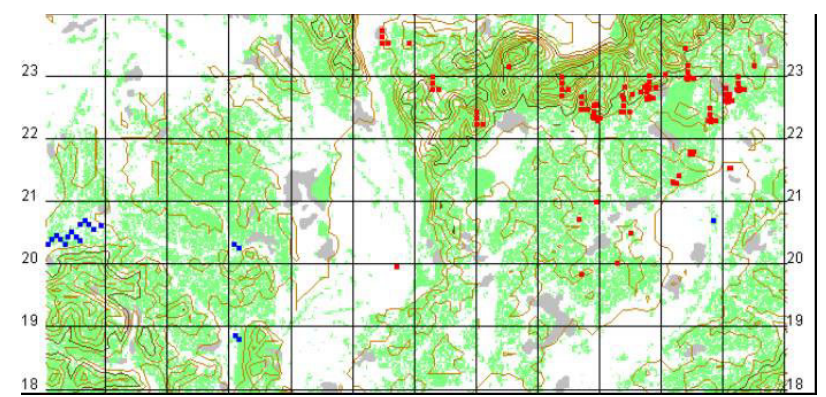

Figure 3: Experimental Scenario
This experiment investigates three different treatments for command and control. In the most restrictive treatment for command and control, no tactical decision agents are used. Friendly forces must navigate along pre-determined routes to their objectives. This is analogous to very detailed command-by-plan methods of control. In the next treatment, unit agents are used. This allows platoon-sized formations to deviate from prescribed routes and positions in order to best accomplish assigned tasks. This is analogous to Auftragstaktik or directive control where platoon leaders are granted some tactical freedom of action within the accomplishment of assigned tasks. In the least restrictive command and control treatment, both unit agents and vehicle agents are used. Vehicles are permitted to break formation in order to better accomplish assigned tasks. This is analogous to Auftragstaktik where both platoon and vehicle commanders are granted tactical freedom of action within the accomplishment of assigned tasks.

The experiment investigates two treatments for force design. The simulated capabilities of the base force design are roughly similar to those of current US Army heavy forces equipped with tanks and infantry fighting vehicles. For the second force design, friendly forces had capabilities roughly similar to those expected of the Army Future Force equipped with the Future Combat System (FCS) (TRADOC Pamphlet 525-3-90, 2002). These forces had responsive precision fires, improved reconnaissance and surveillance systems, but less armor than the current forces. (A disclaimer is necessary here. The data used to adjudicate combat was unclassified and not from an official source. JCombat has not been accredited by any US Army authority. The purpose of this experiment was to test the ability of a simulation to model command and control. It is not intended to be a rigorous comparison between current and future forces.) JCombat ran for 20 iterations for each combination of command and control and force design for a total of 120 runs.

\section{EXPERIMENTAL RESULTS}

Tables 1 and 2 summarize the performance of friendly forces for each of the treatments. These results are also graphed in Figures 4 and 5.

Table 1: Friendly Losses

\begin{tabular}{|c|l|r|r|}
\hline & & \multicolumn{2}{|c|}{ Force } \\
\hline Agents & Data & Base & FCS \\
\hline \multirow{2}{*}{ None } & Average of Friendly Losses & 21.5 & 14.1 \\
\cline { 2 - 4 } & StdDev of Friendly Losses & 4.0 & 3.5 \\
\hline \multirow{2}{*}{ Unit } & Average of Friendly Losses & 19.8 & 15.0 \\
\cline { 2 - 4 } & StdDev of Friendly Losses & 3.0 & 2.8 \\
\hline \multirow{2}{*}{ Vehicle } & Average of Friendly Losses & 19.5 & 12.3 \\
\cline { 2 - 4 } & StdDev of Friendly Losses & 3.3 & 3.3 \\
\hline
\end{tabular}

Inspection of these results shows the effect of less restrictive command and control. For friendly losses, command and control had no significant effect for either force 
Table 2: Enemy Losses

\begin{tabular}{|c|l|r|r|}
\hline & & \multicolumn{2}{|c|}{ Force } \\
\hline Agents & Data & Base & FCS \\
\hline \multirow{2}{*}{ None } & Average of Enemy Losses & 27.7 & 46.0 \\
\cline { 2 - 4 } & StdDev of Enemy Losses & 9.0 & 6.1 \\
\hline \multirow{2}{*}{ Unit } & Average of Enemy Losses & 33.9 & 56.3 \\
\cline { 2 - 4 } & StdDev of Enemy Losses & 14.4 & 11.4 \\
\hline \multirow{2}{*}{ Vehicle } & Average of Enemy Losses & 41.1 & 84.9 \\
\cline { 2 - 4 } & StdDev of Enemy Losses & 14.4 & 13.3 \\
\hline
\end{tabular}

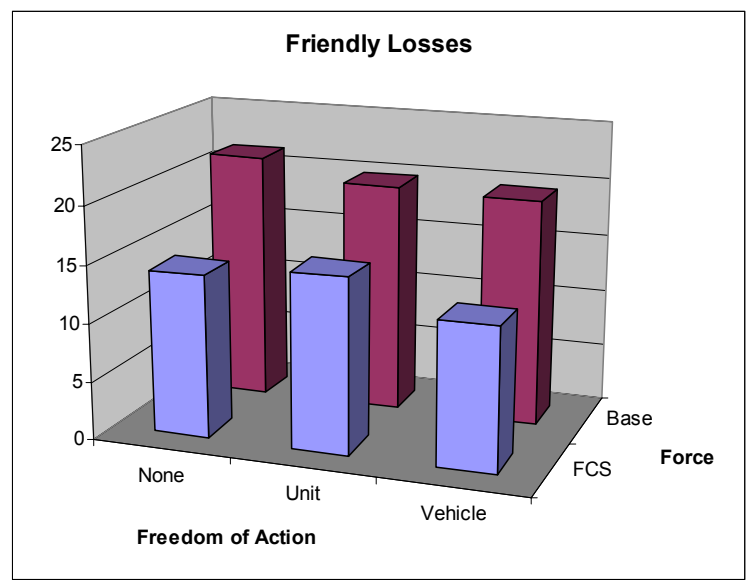

Figure 4: Friendly Losses

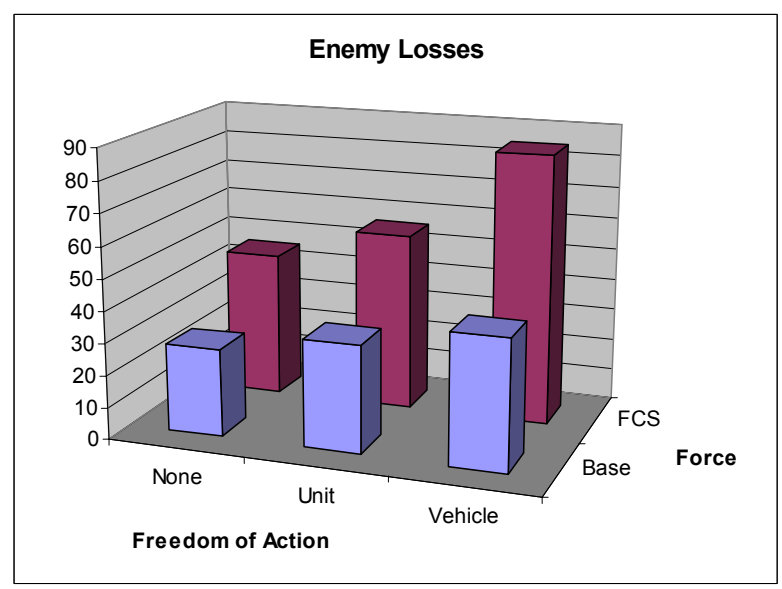

Figure 5: Enemy Losses

design. For enemy losses, movement to less restrictive command and control led to significant increases in enemy losses for each force design. Furthermore, there was a very strong interaction between force design and command and control. For the future forces, Auftragstaktik provided much greater payoff than it did for the base case forces.

\section{CONCLUSIONS AND FUTURE WORK}

Three interesting conclusions follow from the experiment described in this paper. First, tactical decision agents have demonstrated an ability to estimate the effects of command and control in a tactical simulation model. This is a critical capability for modeling the effects of information-enabled forces. Second, more freedom of action, or Auftragstaktik, led to better performance by friendly forces. Finally, the effects of Auftragstaktik were significantly greater for future forces than they were for current forces.

These simulation results add objective evidence to Major General Widder's claim that "only Auftragstaktik" enables exploitation of sophisticated technology. As tactical forces have more information and more capability at lower tactical levels, commanders must allow those forces freedom of action to exploit those capabilities within the framework of their intent. A fundamental change in command and control is required to leverage the information advantage of future forces. Given that change, the commander's job is simpler in the information age, but he is more effective. He spends more time on a small number of critical decisions as opposed to internal coordination and synchronization. To change the plan, he only needs to communicate a change in intent.

There is fruitful ground for further experimentation with tactical decision agents. The first critical task is to integrate decision agents into existing tactical simulations. Also, different simulations and different tactical entities call for different heuristics for these decision agents. Once these agents have been improved and embedded in more robust simulations, analysts will have the ability to estimate the effects of additional command and control variables. These include quality of information, timeliness of information, autonomy, trust, force capability, and agent capability. These experiments will provide insight into the design and employment of future combat forces.

\section{REFERENCES}

German Army Regulation (AR) 100/100. 1998. (Restricted). Army Command and Control (Bonn, GE: 15 October 1998): 302.

Ilachinski, A. 1996. Land Warfare and Complexity, Part I: Mathematical Background and Technical Sourcebook, Alexandria, VA: Center for Naval Analysis, July 1996, 101-102.

Kewley, R. and L. Larimer. 2003. An Agent-BasedModeling Approach to Quantifying the Value of Batllefield Information. Phalanx V 36(2): 10-14, 25-26.

TRADOC Pamphlet 525-3-90. 2002. Objective Force Maneuver Units of Action. United States Army Training and Doctrine Command, 1 November 2002.

Widder, W. 2002. Auftragstaktik and Innere Fuhrung: Trademarks of German Leadership. Military Review V 82(5): 3-9.

\section{AUTHOR BIOGRAPHIES}

MAJOR ROBERT KEWLEY currently serves as an operational capabilities analyst at the Center for Army Analy- 
sis. He has analysis or research experience in the areas of theater campaign modeling, combat identification, C4ISR modeling, and agent-based models. He holds a M.S. in Industrial and Managerial Engineering and a Ph.D. in Decision Science and Engineering Systems from Rensselaer Polytechnic Institute. His e-mail address is <robert. kewley@us.army.mil>. 